\title{
Statistical Learning Procedure in Loopy Belief Propagation for Probabilistic Image Processing
}

\author{
Kazuyuki Tanaka \\ Graduate School of Information Sciences, Tohoku University \\ Aramaki-aza-aoba 6-3-09, Aoba-ku, Sendai 980-8579, Japan \\ e-mail: kazu@smapip.is.tohoku.ac.jp \\ webpage: http://www.smapip.is.tohoku.ac.jp/kazu/
}

\begin{abstract}
We give a fast and practical algorithm for statistical learning hyperparameters from observable data in probabilistic image processing, which is based on Gaussian graphical model and maximum likelihood estimation. Although hyperparameters in the probabilistic model are determined so as to maximize a marginal likelihood, a practical algorithm is described for the EM algorithm with the loopy belief propagation which is one of approximate inference algorithms in artificial intelligence.
\end{abstract}

\section{Introduction}

In the area of artificial intelligence, belief propagation has been investigated as a tool for probabilistic inference[1]. In computer sciences, the methodologies for estimation of the hyperparameters only from the observable data have been investigated as statistical learning theory[2]. As one of the criteria for determining hyperparameters, we have the maximization of marginal likelihood[3] It is known that the expectation-maximization (EM) algorithm in the maximum likelihood method is a powerful method for estimation of hyperparameters[3] and can provide us a fast algorithm to achieve a statistical learning system.

The loopy belief propagation has been studied for some practical applications of the image processing $[5,6]$. Tanaka et al [4] have investigated the accuracy of loopy belief propagation for the estimation of hyperparameters by means of the maximization of marginal likelihood from observable data in probabilistic image processing. They calculated the marginal likelihood itself to determine the hyperparameters and spent about four hours as computational time in a personal computer for estimating hyperparameters in a graylevel image with $256 \times 256$ pixels. By applying the EM algorithm to the determination of hyperparameters, it is ex- pected to achieve a fast and practical algorithm for statistical learning of hyperparameters from the observable data in the probabilistic image processing.

Our main purpose in the present paper is to give statistical learning system of the EM algorithm for the maximization of marginal likelihood by means of the loopy belief propagation in gray-level image restorations.

\section{Probabilistic Image Restorations based on Gaussian Graphical Model and EM algo- rithm}

In this section, we give the basic framework of the probabilistic image restoration and the hyperparameter estimation by the EM algorithm. The a priori probabilistic model is assumed to be the Gaussian graphical model and the degradation process is supposed to be the additive white Gaussian noise.

In computer vision, images are typically defined on a set of points arranged on a rectangular lattice. Each point is called a pixel. At each pixel, the intensity of light is represented as an integer or a real number. We consider an image on a square lattice $\Omega \equiv\{i\}$ such that the intensity at each pixel takes a real value in the range $(-\infty,+\infty)$. A monochrome digital image is then expressed as a twodimensional light intensity function $f_{i}$, where $f_{i}$ is proportional to the brightness of the image at the pixel $i$. The square lattice $\Omega$ is assumed to have periodic boundary conditions in both vertical and horizontal directions. The intensities at pixel $i$ in the original image and the degraded image are regarded as random variables denoted by $F_{i}$ and $G_{i}$, respectively, and the random fields of intensities in the original image and the degraded image are represented by $\vec{F} \equiv\left\{F_{i} \mid i \in \Omega\right\}$ and $\vec{G} \equiv\left\{G_{i} \mid i \in \Omega\right\}$, respectively. The actual original image and degraded image are denoted by $\vec{f}=\left\{f_{i} \mid i \in \Omega\right\}$ and $\vec{g}=\left\{g_{i} \mid i \in \Omega\right\}$, respectively.

The probability density for the original image $\vec{f}, \mathcal{P}(\vec{F}=$ 
$\vec{f})$, is called the a priori probability density of the image. As a consequence of Bayes' formula, the a posteriori probability density $\mathcal{P}(\vec{F}=\vec{f} \mid \vec{G}=\vec{g})$, that the original image is $\vec{f}$ when the given degraded image is $\vec{g}$, is expressed as

$\mathcal{P}(\vec{F}=\vec{f} \mid \vec{G}=\vec{g})=\frac{\mathcal{P}(\vec{G}=\vec{g} \mid \vec{F}=\vec{f}) \mathcal{P}(\vec{F}=\vec{f})}{\int \mathcal{P}(\vec{G}=\vec{g} \mid \vec{F}=\vec{z}) \mathcal{P}(\vec{F}=\vec{z}) d \vec{z}}$,

where $\int d \vec{z} \equiv \prod_{i \in \Omega} \int_{-\infty}^{+\infty} d z_{i}$. The probability $\mathcal{P}(\vec{G}=$ $\vec{g} \mid \vec{F}=\vec{f})$ is the conditional probability density that the degraded image is $\vec{g}$ when the original image is $\vec{f}$, and it describes the degradation process.

In the present paper, it is assumed that the degraded image $\vec{g}$ is generated from the original image $\vec{f}$ by the addition of white Gaussian noise with mean 0 and variance $\sigma^{2}$ $(\sigma>0)$, so that

$$
\begin{aligned}
& \mathcal{P}(\vec{G}=\vec{g} \mid \vec{F}=\vec{f}, \sigma) \\
& \quad \equiv\left(\frac{1}{2 \pi \sigma^{2}}\right)^{\frac{|\Omega|}{2}} \prod_{i \in \Omega} \exp \left(-\frac{1}{2 \sigma^{2}}\left(f_{i}-g_{i}\right)^{2}\right)
\end{aligned}
$$

where $|\Omega|$ is the total number of pixels ${ }^{1}$. Moreover, the $a$ priori probability density that the original image is $\vec{f}$ is assumed to be

$$
\mathcal{P}(\vec{F}=\vec{f} \mid \alpha) \equiv \frac{\prod_{i j \in \mathcal{N}} \exp \left(-\frac{1}{2} \alpha\left(f_{i}-f_{j}\right)^{2}\right)}{\int \prod_{i j \in \mathcal{N}} \exp \left(-\frac{1}{2} \alpha\left(f_{i}-f_{j}\right)^{2}\right) d \vec{z}},
$$

which represents local spatial correlation among the pixel intensities. The notation $\mathcal{N}$ is the set of all the nearestneighbor pairs of pixels on the square lattice $\Omega$. By substituting Eqs.(2) and (3) into Eq.(1), we obtain

$$
\begin{aligned}
& \mathcal{P}(\vec{F}=\vec{f} \mid \vec{G}=\vec{g}, \alpha, \sigma) \\
& =\frac{1}{\mathcal{Z}} \exp \left(-\frac{1}{2 \sigma^{2}} \sum_{i \in \Omega}\left(f_{i}-g_{i}\right)^{2}-\frac{1}{2} \alpha \sum_{i j \in \mathcal{N}}\left(f_{i}-f_{j}\right)^{2}\right) .
\end{aligned}
$$

Here $\mathcal{Z}$ is the normalization constant.

In the maximum likelihood approach, values for the hyperparameters $\alpha$ and $\sigma$ are determined so as to maximize the marginal likelihood $\mathcal{P}(\vec{G}=\vec{g} \mid \alpha, \sigma)$, where

$$
\begin{aligned}
& \mathcal{P}(\vec{G}=\vec{g} \mid \alpha, \sigma) \\
& \quad \equiv \int \mathcal{P}(\vec{G}=\vec{g} \mid \vec{F}=\vec{z}, \sigma) \mathcal{P}(\vec{F}=\vec{z} \mid \alpha) d \vec{z} .
\end{aligned}
$$

We denote these maximizers by $\widehat{\alpha}$ and $\widehat{\sigma}$ :

$$
(\widehat{\alpha}, \widehat{\sigma})=\arg \max _{(\alpha, \sigma)} \mathcal{P}(\vec{G}=\vec{g} \mid \alpha, \sigma) .
$$

\footnotetext{
${ }^{1}$ We denote the number of whole elements belonging to a set $A$ by $|A|$
}

Given the estimates $\widehat{\alpha}$ and $\widehat{\sigma}$, the restored image $\vec{h}=$ $\left\{h_{i} \mid i \in \Omega\right\}$ is determined by

$$
h_{i} \equiv \int z_{i} \mathcal{P}(\vec{F}=\vec{z} \mid \vec{G}=\vec{g}, \widehat{\alpha}, \widehat{\sigma}) d \vec{z} .
$$

It is known that the maximization of marginal likelihood in Eq.(6) can be achieved by means of the EM algorithm, which is one of familiar techniques in the statistical learning theory. By introducing the following function:

$$
\begin{aligned}
& \mathcal{Q}\left(\alpha, \sigma \mid \alpha^{\prime}, \sigma^{\prime}, \vec{g}\right) \\
& \equiv \int \mathcal{P}\left(\vec{F}=\vec{z} \mid \vec{G}=\vec{g}, \alpha^{\prime}, \sigma^{\prime}\right) \\
& \quad \times \ln (\rho(\vec{F}=\vec{z}, \vec{G}=\vec{g} \mid \alpha, \sigma)) d \vec{z}
\end{aligned}
$$

the EM algorithm is summarized as follows:

\section{EM Algorithm}

Step 1: $\operatorname{Set}(\alpha(0), \sigma(0))$ and $t \leftarrow 0$.

Step 2: Iterate the following EM step until convergence: $(\alpha(t+$ $1), \sigma(t+1))$ as follows:

$$
\begin{aligned}
& (\alpha(t+1), \sigma(t+1)) \\
& \quad \leftarrow \arg \max _{(\alpha, \sigma)} \mathcal{Q}(\alpha, \sigma \mid \alpha(t), \sigma(t), \vec{g}),
\end{aligned}
$$

and $t \leftarrow t+1$.

\section{Loopy Belief Propagation}

In this section, we present the loopy belief propagation for probabilistic models of the general form

$$
\rho(\vec{f} \mid \vec{g}, \alpha, \beta)=\frac{\prod_{i j \in \mathcal{N}} \phi_{i j}\left(f_{i}, f_{j}\right)}{\int \prod_{i j \in \mathcal{N}} \phi_{i j}\left(z_{i}, z_{j}\right) d \vec{z}}
$$

where

$$
\begin{aligned}
\phi_{i j}\left(\xi, \xi^{\prime}\right) & \equiv \exp \left(-\frac{1}{8} \beta\left(\xi-g_{i}\right)^{2}\right. \\
& \left.-\frac{1}{8} \beta\left(\xi^{\prime}-g_{j}\right)^{2}-\frac{1}{2} \alpha\left(\xi-\xi^{\prime}\right)^{2}\right) .
\end{aligned}
$$

For the loopy belief propagation, we introduce two types of marginal probability densities, defined by

$$
\rho_{i}(\xi \mid \vec{g}, \alpha, \beta) \equiv \int \delta\left(\xi-z_{i}\right) \rho(\vec{z} \mid \vec{g}, \alpha, \beta) d \vec{z},
$$

$$
\begin{aligned}
& \rho_{i j}\left(\xi, \xi^{\prime} \mid \vec{g}, \alpha, \beta\right)=\rho_{j i}\left(\xi^{\prime}, \xi \mid \vec{g}, \alpha, \beta\right) \\
& \quad \equiv \int \delta\left(\xi-z_{i}\right) \delta\left(\xi^{\prime}-z_{j}\right) \rho(\vec{z} \mid \vec{g}, \alpha, \beta) d \vec{z}
\end{aligned}
$$


For consistency, these probability densities should satisfy

$$
\rho_{i}(\xi \mid \vec{g}, \alpha, \beta)=\int_{-\infty}^{+\infty} \rho_{i j}(\xi, \zeta \mid \vec{g}, \alpha, \beta) d \zeta \quad\left(j \in \mathcal{N}_{i}\right) .
$$

Here the notation $\mathcal{N}_{i} \equiv\{j \mid i j \in \mathcal{N}\}$ represents the set of all the nearest-neighbor pairs of pixel $i$. The marginal probability densities can be given approximately by expressions of the forms

$$
\begin{gathered}
\rho_{i}(\xi \mid \vec{g}, \alpha, \beta) \simeq \frac{1}{\mathcal{Z}_{i}} \prod_{k \in \mathcal{N}_{i}} \mathcal{M}_{k \rightarrow i}(\xi), \\
\rho_{i j}\left(\xi, \xi^{\prime} \mid \vec{g}, \alpha, \beta\right) \simeq \frac{1}{\mathcal{Z}_{i j}}\left(\prod_{k \in \mathcal{N}_{i} \backslash j} \mathcal{M}_{k \rightarrow i}(\xi)\right) \\
\times \phi_{i j}\left(\xi, \xi^{\prime}\right)\left(\prod_{k^{\prime} \in \mathcal{N}_{j} \backslash i} \mathcal{M}_{k^{\prime} \rightarrow j}\left(\xi^{\prime}\right)\right) .
\end{gathered}
$$

Here $\mathcal{Z}_{i}$ and $\mathcal{Z}_{i j}$ are the normalization constants of $\rho_{i}(\xi \mid \vec{g}, \alpha, \beta)$ and $\rho_{i j}\left(\xi, \xi^{\prime} \mid \vec{g}, \alpha, \beta\right)$, respectively. In probabilistic inference, the quantity $\mathcal{M}_{k \rightarrow i}(\xi)$ is referred to as a message propagated from $k$ to $i$. If we substitute Eqs. (15) and (16) into Eq. (14), the deterministic equations for $\mathcal{M}_{k \rightarrow i}(\xi)$ can be derived as

$$
\begin{aligned}
& \mathcal{M}_{j \rightarrow i}(\xi) \\
& =\frac{\int_{-\infty}^{+\infty} \phi_{i j}\left(\xi, \xi^{\prime}\right) \prod_{k \in \mathcal{N}_{j} \backslash i} \mathcal{M}_{k \rightarrow j}\left(\xi^{\prime}\right) d \xi^{\prime}}{\int_{-\infty}^{+\infty} \int_{-\infty}^{+\infty} \phi_{i j}\left(\xi^{\prime \prime}, \xi^{\prime}\right) \prod_{k \in \mathcal{N}_{j} \backslash i} \mathcal{M}_{k \rightarrow j}\left(\xi^{\prime}\right) d \xi^{\prime} d \xi^{\prime \prime}}
\end{aligned}
$$

Now we assume that $\mathcal{M}_{j \rightarrow i}(\xi)$ can be approximately expressed as

$$
\mathcal{M}_{j \rightarrow i}(\xi) \simeq \sqrt{\frac{\lambda_{j \rightarrow i}}{2 \pi}} \exp \left(-\frac{\lambda_{j \rightarrow i}}{2}\left(\xi-\mu_{j \rightarrow i}\right)^{2}\right) .
$$

The simultaneous fixed-point equation (17) can be reduced to the following fixed point equations for $\left\{\lambda_{j \rightarrow i}, \mu_{j \rightarrow i} \mid j \in \mathcal{N}_{i}, i \in \Omega\right\}$ :

$$
\begin{aligned}
\frac{1}{\lambda_{j \rightarrow i}} & =\frac{1}{\alpha}+\frac{1}{\beta+\sum_{k \in \mathcal{N}_{j} \backslash i} \lambda_{k \rightarrow j}}, \\
\mu_{j \rightarrow i} & =\frac{\beta g_{j}+\sum_{k \in \mathcal{N}_{j} \backslash i} \mu_{k \rightarrow j} \lambda_{k \rightarrow j}}{\beta+\sum_{k \in \mathcal{N}_{j} \backslash i} \lambda_{k \rightarrow j}} .
\end{aligned}
$$

If we substitute Eq. (18) into Eqs. (15) and (16), the oneand two-body marginal probability densities $\rho_{i}(\xi \mid \vec{g}, \alpha, \beta)$ and $\rho_{i j}\left(\xi, \xi^{\prime} \mid \vec{g}, \alpha, \beta\right)$ are approximately obtained. The simultaneous fixed-point equations (19) and (20) are solved by the following iterative algorithm.

Algorithm LBP $[\vec{g}, \alpha, \beta]$

Step 1: Set $r \leftarrow 0$ as an initial value.

Step 2: Update $r \leftarrow r+1$ and

$$
\begin{aligned}
& a_{j \rightarrow i}(r) \leftarrow\left(\frac{1}{\alpha}+\frac{1}{\beta+\sum_{k \in \mathcal{N}_{j} \backslash i} a_{k \rightarrow j}(r-1)}\right)^{-1}, \\
& b_{j \rightarrow i}(r) \leftarrow \frac{\beta g_{j}+\sum_{k \in \mathcal{N}_{j} \backslash i} b_{k \rightarrow j}(r-1) a_{k \rightarrow j}(r-1)}{\beta+\sum_{k \in \mathcal{N}_{j} \backslash i} a_{k \rightarrow j}(r-1)} .
\end{aligned}
$$

Step 3: Update $R \leftarrow r, \quad \lambda_{j \rightarrow i} \leftarrow a_{j \rightarrow i}(r)$ and $\mu_{j \rightarrow i} \leftarrow b_{j \rightarrow i}(r)$ $(i \in \Omega)$. Go to Step 4 if, for prespecified $\varepsilon$,

$$
\begin{aligned}
\sum_{i \in \Omega} \sum_{j \in \mathcal{N}_{i}} & \left(\left|a_{j \rightarrow i}(r)-a_{j \rightarrow i}(r-1)\right|\right. \\
+ & \left.\left|b_{j \rightarrow i}(r)-b_{j \rightarrow i}(r-1)\right|\right)<\varepsilon,
\end{aligned}
$$

and go to Step 2 otherwise.

Step 4: Substitute $\left\{\lambda_{j \rightarrow i}, \mu_{j \rightarrow i} \mid j \in \mathcal{N}_{i}, i \in \Omega\right\}$ into Eq. (18), and calculate $\rho_{i}(\xi \mid \vec{g}, \alpha, \beta)$ and $\rho_{i j}\left(\xi, \xi^{\prime} \mid \vec{g}, \alpha, \beta\right)$ by using Eqs.(15) and (16). Stop after $\rho_{i}(\xi \mid \vec{g}, \alpha, \beta)$ and $\rho_{i j}\left(\xi, \xi^{\prime} \mid \vec{g}, \alpha, \beta\right)$ are set as outputs in the present algorithm.

Again, it is usually adequate to set $\varepsilon=10^{-6}$. In the denominators of Eqs. (21) and (22), the summations $\sum_{k \in \mathcal{N}_{j} \backslash i} a_{k \rightarrow j}(r-1)$ and $\sum_{k \in \mathcal{N}_{j} \backslash i} b_{k \rightarrow j}(r-1) a_{k \rightarrow j}(r-1)$ can be evaluated in $\mathcal{O}(1)$ time per pair of pixels $i$ and $j$, because the number of elements in the set $\mathcal{N}_{j} \backslash i$ is equal to 3 per pair of pixels. Hence the iterative algorithm for solving the simultaneous fixed-point equations (19) and (20) requires a total of $\mathcal{O}(|\Omega|)$ computations per update.

For fixed values of $\alpha^{\prime}$ and $\sigma^{\prime}$, the extremum conditions of $Q\left(\alpha, \sigma \mid \alpha^{\prime}, \sigma^{\prime}, \vec{g}\right)$ with respect to $\sigma$ and $\alpha$ are reduced to the following equations:

$$
\begin{gathered}
\sum_{i \in \Omega} \int_{-\infty}^{+\infty}\left(\xi-g_{i}\right)^{2} \rho_{i}\left(\xi \mid \vec{g}, \alpha^{\prime}, \sigma^{\prime-2}\right) d \xi=|\Omega| \sigma^{2} \\
\sum_{i j \in \mathcal{N}} \int_{-\infty}^{+\infty} \int_{-\infty}^{+\infty}\left(\xi-\xi^{\prime}\right)^{2} \rho_{i j}\left(\xi, \xi^{\prime} \mid \vec{g}, \alpha^{\prime}, \sigma^{\prime-2}\right) d \xi d \xi^{\prime} \\
=\sum_{i j \in \mathcal{N}} \int_{-\infty}^{+\infty} \int_{-\infty}^{+\infty}\left(\xi-\xi^{\prime}\right)^{2} \rho_{i j}\left(\xi, \xi^{\prime} \mid \vec{g}, \alpha, 0\right) d \xi d \xi^{\prime},
\end{gathered}
$$


respectively. The marginal probability density function $\rho_{i}\left(\xi \mid \vec{g}, \alpha^{\prime}, \sigma^{\prime-2}\right), \quad \rho_{i j}\left(\xi, \xi^{\prime} \mid \vec{g}, \alpha^{\prime}, \sigma^{\prime-2}\right) \quad$ and $\rho_{i j}\left(\xi, \xi^{\prime} \mid \vec{g}, \alpha^{\prime}, 0\right)$ are approximately obtained as outputs of the loopy belief propagation algorithms $\operatorname{LBP}\left[\vec{g}, \alpha^{\prime}, \sigma^{\prime-2}\right]$ and $\operatorname{LBP}[\vec{g}, \alpha, 0]$. Therefore, we can give the EM algorithm for the maximization of marginal likelihood in Eq.(6) by using the loopy belief propagation as follows:

\section{EM Algorithm in Loopy Belief Propagation}

Step 1: $\operatorname{Set}(\alpha(0), \sigma(0))$ and $t \leftarrow 0$.

Step 2: Run the algorithm $\operatorname{LBP}\left[\vec{g}, \alpha(t), \sigma(t)^{-2}\right]$ and update $\sigma(t)$ and $A$ as follows:

$$
\sigma(t+1) \leftarrow \frac{1}{|\Omega|} \sum_{i \in \Omega} \int_{-\infty}^{+\infty}\left(\xi-g_{i}\right)^{2} \rho_{i}\left(\xi \mid \vec{g}, \alpha(t), \sigma(t)^{-2}\right) d \xi,
$$

$$
\begin{aligned}
A \leftarrow \sum_{i j \in \mathcal{N}} \int_{-\infty}^{+\infty} \int_{-\infty}^{+\infty}\left(\xi-\xi^{\prime}\right)^{2} \\
\quad \times \rho_{i j}\left(\xi, \xi^{\prime} \mid \vec{g}, \alpha(t), \sigma(t)^{-2}\right) d \xi d \xi^{\prime}
\end{aligned}
$$

Step 3: Run the algorithm $\operatorname{LBP}[\vec{g}, \alpha, 0]$ for various positive values of $\alpha$ and set $\alpha(t+1)$ to the value of $\alpha$ which satisfies the follows equation:

$$
\sum_{i j \in \mathcal{N}} \int_{-\infty}^{+\infty} \int_{-\infty}^{+\infty}\left(\xi-\xi^{\prime}\right)^{2} \rho_{i j}\left(\xi, \xi^{\prime} \mid \vec{g}, \alpha, 0\right) d \xi d \xi^{\prime}=A,
$$

and $t \leftarrow t+1$.

Step 4: Update

$$
(\widehat{\alpha}, \widehat{\sigma}) \leftarrow(\alpha(t), \sigma(t)) .
$$

Stop if the values of $\widehat{\alpha}$ and $\widehat{\sigma}$ converge, and return to Step 2 otherwise.

\section{Numerical Experiments}

In this section, we present the results of some numerical experiments for the restoration of gray-level images. The optimal values for the hyperparameters, $(\widehat{\alpha}, \widehat{\sigma})$, are determined by means of EM algorithm in the loopy belief propagation.

In the present paper, our framework for probabilistic image restoration uses the Gaussian graphical model and considers a continuous random variable for the intensity at each pixel in the original and the degraded images. However, in practical images in computer vision, the intensity of light at each pixel is represented as an integer chosen from the set $\{0,1, \cdots, 255\}$. In our numerical experiments, we apply the

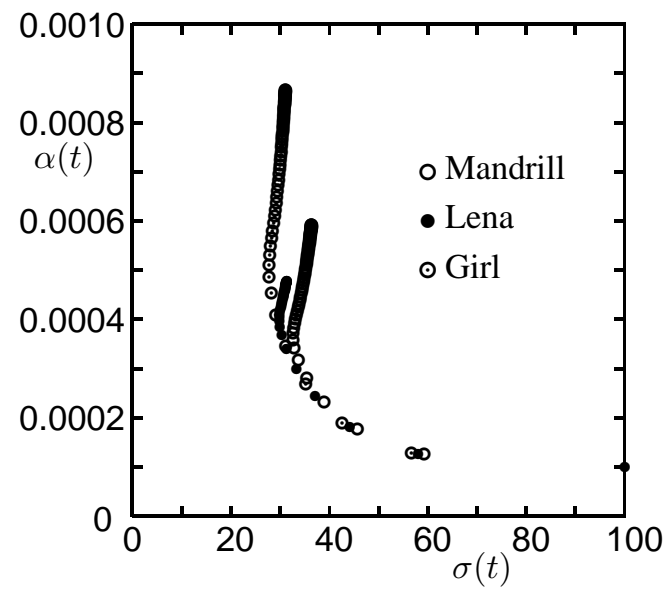

Figure 1. The dynamical behaviours of the iterative process in the EM algorithm in the loopy belief propagation.

framework established in the previous sections to images consisting of integers $\{0,1, \cdots, 255\}$. Instead of Eq.(7), we use

$$
h_{i} \leftarrow \arg \min _{n=0,1, \cdots, 255}\left(n-\int \xi \rho_{i}(\xi \mid \vec{g}, \widehat{\alpha}, \widehat{\sigma}) d \xi\right)^{2} .
$$

We then performed numerical experiments based on the standard images "Lena", "Girl" and "Mandrill". Degraded images $\vec{g}$ are produced from the original images $\vec{f}$ with $\sigma=40$. By using the EM algorithm in the loopy belief propagation, we achieve the maximization of the marginal likelihood (6). For the degraded images $\vec{g}$ produced from the standard images "Lena", "Girl" and "Mandrill", the dynamical behaviours for the iterative procedure of the EM algorithm in the loopy belief propagation are given in Fig. 1. The image restorations created by means of the loopy belief propagation for the Gaussian graphical model are shown in Figs. 2-4. We give in Table 1 the estimates, $\widehat{\sigma}$ and $\widehat{\alpha}$, of the hyperparameters, and the values of the mean-squared error $d(\vec{f}, \vec{h}) \equiv \frac{1}{\Omega} \sum_{i \in \Omega}\left(f_{i}-h_{i}\right)^{2}$ and the improvement in the signal to noise ratio, $\Delta_{\mathrm{SNR}} \equiv 10 \log _{10}\left(\frac{\sum_{i \in \Omega}\left(g_{i}-f_{i}\right)^{2}}{\sum_{i \in \Omega}\left(\widehat{f}_{i}-f_{i}\right)^{2}}\right)$ in decibels $(\mathrm{dB})$.

\section{Concluding Remarks}

In this paper, we have studied the statistical learning system of the hyperparameter in the probabilistic image processing based on the Gaussian graphical model and the loopy belief propagation. The estimates of the hyperparameters are determined so as to maximize the marginal likelihood. The main purpose of the present paper is to construct the fast and practical algorithm for the hyperparameter estimation based on the EM algorithm of the loopy belief 
(a)

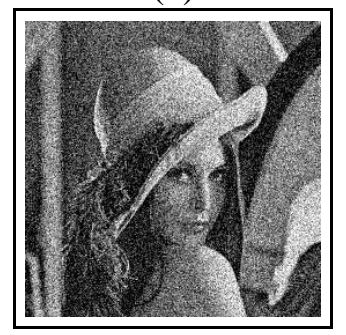

(b)

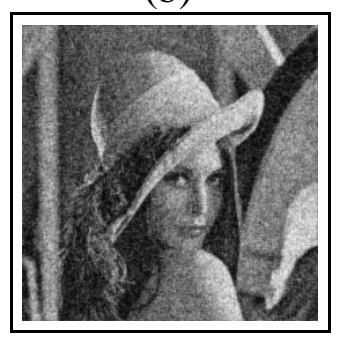

Figure 2. Image Restoration for the standard image "Lena" by the EM algorithm in the loopy belief propagation. (a) Degraded image $\vec{g}$ produced by the additive white Gaussian noise with the mean 0 and the variance $40^{2}$. (b) Restored image $\vec{h}$ by the loopy belief propagation and the EM algorithm.

Table 1. Estimates of hyperparameters, $\widehat{\alpha}$ and $\widehat{\sigma}$, and the values of $d(\vec{f}, \vec{h})$ and $\Delta_{\mathrm{SNR}}$ (dB) obtained by using the belief propagation. The degraded images $\vec{g}$ are produced for $\sigma=40$.

\begin{tabular}{ccccc}
\hline & $\widehat{\alpha}$ & $\widehat{\sigma}$ & $d(\vec{f}, \vec{h})$ & $\Delta_{\text {SNR }}(\mathrm{dB})$ \\
\hline Lena & 0.000478 & 31.32 & 328 & 6.360 \\
Girl & 0.000866 & 31.09 & 199 & 8.013 \\
Mandrill & 0.000592 & 36.38 & 330 & 6.654 \\
\hline
\end{tabular}

propagation. The EM algorithm with the loopy belief propagation can give us the smooth dynamical behaviour in the iterative procedure for the EM algorithm. For a gray-level image with $256 \times 256$ size, our proposed EM algorithm with the loopy belief propagation spent about five minutes as the computational time in the personal computer SONY VAIO VGN-T70B, while it has taken about four hours as computational time in the same personal computer for maximizing the marginal likelihood directly by means of the loopy belief propagation.

We have treated only the probabilistic image processing based on the Gaussian graphical model in the present paper. However, we have to adopt a different probabilistic model depending on the purpose of image processing [5, 6]. Moreover, some generalizations of the loopy belief propagation have been done[7, 8]. The present author have studied the accuracy of a generalized belief propagation in the statistical learning system in the hyperparameters for the Gaussian graphical model[9]. It is a future problem to extend the loopy belief propagation to more general and practical algorithms in the EM algorithm of the statistical learning (a)

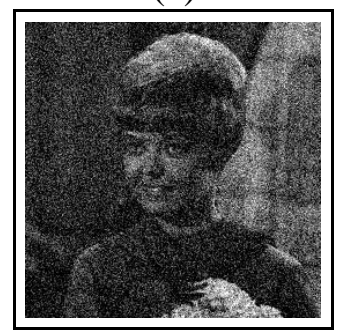

(b)

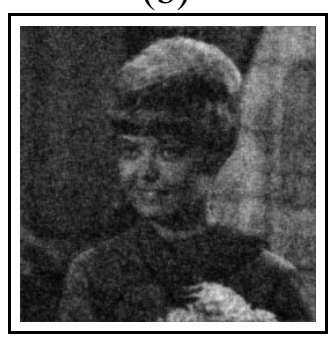

Figure 3. Image Restoration for the standard image "Girl" by the EM algorithm in the loopy belief propagation. (a) Degraded image $\vec{g}$ produced by the additive white Gaussian noise with the mean 0 and the variance $40^{2}$. (b) Restored image $\vec{h}$ by the loopy belief propagation and the EM algorithm.

systems.

\section{Acknowledgements}

The authors are grateful to Professor D. M. Titterington of the Department of Statistics, University of Glasgow, for valuable discussions. This work was partly supported by the Grants-In-Aid (No.14084101, No.14084203 and No.17500134) for Scientific Research from the Ministry of Education, Culture, Sports, Science and Technology of Japan.

\section{References}

[1] J. Pearl, Probabilistic Reasoning in Intelligent Systems: Networks of Plausible Inference Morgan Kaufmann, 1988.

[2] M. I. Jordan (eds): Learning in Graphical Models, MIT Press, Cambridge, 1999.

[3] J. Zhang, "The mean field theory in EM procedures for Markov Random Fields," IEEE Transactions on Signal Processing, vol.40, no.10, pp.2570-2583, 1992.

[4] K. Tanaka, H. Shouno, M. Okada and D. M. Titterington, "Accuracy of the Bethe approximation for hyperparameter estimation in probabilistic image processing," J. Phys. A: Math. \& Gen., vol.37, no.36, pp.8675-8696, 2004.

[5] W. T. Freeman, T. R. Jones and E. C. Pasztor, "Example-based super-resolution," IEEE Computer 
(a)

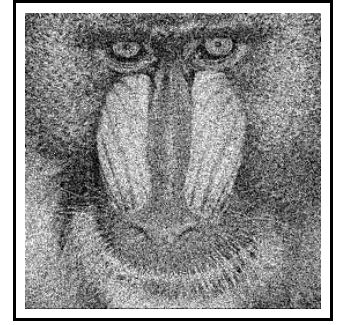

(b)

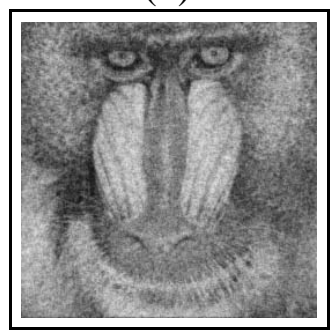

Figure 4. Image Restoration for the standard image "Mandrill" by the EM algorithm in the loopy belief propagation. (a) Degraded image $\vec{g}$ produced by the additive white Gaussian noise with the mean 0 and the variance $40^{2}$. (b) Restored image $\vec{h}$ by the loopy belief propagation and the EM algorithm.

Graphics and Applications, vol.22, no.2, pp.56-65, 2002.

[6] J. Sun, N.-N. Zheng and H.-Y. Shum, "Stereo matching using belief propagation," IEEE Transactions on Pattern Analysis and Machine Intelligence, vol.25, no.7, pp.787-800, 2003.

[7] M. Opper and D. Saad (eds), Advanced Mean Field Methods - Theory and Practice - MIT Press, 2001.

[8] J. S. Yedidia, W. T. Freeman and Y. Weiss: "Constructing free-energy approximations and generalized belief propagation algorithms," IEEE Transactions on Information Theory, vol.51, no.7, pp.2282-2312, 2005.

[9] K. Tanaka, "Generalized Belief Propagation Formula in Probabilistic Information Processing based on Gaussian Graphical Model," submitted to IEICE Transactions D-II. 